\title{
Smart Medicine Drawers Using IOS Application and Arduino Board
}

\author{
Abdulrahman Alkandari, Sarah Alshamery, Samer Moein, Nayfah Mohsen Almutairi \\ Computer Department, Basic Education College (PAAET), Kuwait \\ Aam.alkandari@paaet.edu.kw \\ Computer Department College of Basic Education (PAAET), Kuwait \\ saraalshammery@hotmail.com \\ Electrical and Computer Engineering, University of Victoria, Canada \\ samerm@uvic.ca \\ Islamic Studies Department, Ministry of Awqaf and Islamic Affairs, Kuwait \\ N.almutairi111@alumni.gust.edu.kw
}

\begin{abstract}
As people age and engage with life, many people drop the commitments in things which become like a routine that they go on with There are many patients forget to take their treatment on time, while some of them take the treatment but in irregular schedules, which reduces the effectiveness of the treatment. The method of reminding patients their schedules by the nurse for the elderly and sitter kids is not considered a successful solution in some cases, because it relies on another person, who may probably omit or forgets the exact time. With the proliferation of smartphones, it requires a technology solution to help patients in determining medication schedules accurately and make them more controlling on taking their medication. This paper proposed an application running on the iPhone connecting with smart drawers through the Arduino Board. The primary purpose of this application is to organize and remind patients to take their medicines on the accurate time. Drawers can be opened and closed through the application.
\end{abstract}

Keywords-Blockchain, Consensus algorithm, Performance, Scalability.

\section{INTRODUCTION}

No one can deny that technology has a significant influence on our daily lives. We may disagree with determining whether these effects negatively or positively, but we agree that technology has affected our lives widely and extensively. In recent years we have seen a revolution in smartphones, tablets, and the succession of innovations that have a substantial impact on the pattern of our daily life changes.

One of the most successful treatments of any disease factors is taking medication specific punctual prescribed by a specialist doctor. It is possible to forget taking the dose due to preoccupation with the concerns of life and circumstances. Forgetting any doses of medication reduces the effectiveness of treatment and limiting access to the desired results. For this reason, innovation stimuli electronic reminder of the date to take the drug innovatively. Operate these stimuli to recall and adjust the time according to the time required for the alarm clock which works acoustically or optically or through the application of a mobile phone to send notifications to the users. Thus, it ensures eating their medicine regularly without forgetting any dose of doses. These stimuli are also ideal for patients with Alzheimer's disease or patients who suffer from weakness in memory. For this, the best of these stimuli have been set to help patients in taking their medicines easily without fuss thinking about the timing. [1]

There are many types of operating systems for smartphones. IOS is a system that runs on Apple's iPhone. It has appeared at the beginning of 2007 as the operating system created by Apple for the iPhone. Then the system included iPad tablet devices and iPod Touch.

It was a quantum leap in the world of smartphones. Also, it is the best-known system and that because of the beauty and simplicity of its design, and it is also different from other mobile systems. The IOS system is worthwhile; it has continuous improvements and updates at each period.

\section{PROBLEM STATEMENT}

Everyone has many things that concern them with their time every day. By adding a new task to their full day, people may forget some of the essential things that must be done, such as taking the medications in time. Remembering medications, prescribed and non-prescribed vitamins, is not an easy thing to schedule. This paper presents an effective solution that helps patients to remember their medications daily schedules. 
Many patients think that it is okay to forget taking a few grains of their medications, but this is not the truth. All medicines have the so-called "half-life" by which time their bodies need to get rid of half the dose addressed. When the patients take medication regularly, the medicine in the body up to a state of "stability," which means that the amount of drug absorbed by the body is equal to the amount of medicine they get it out.

Usually, the medication needs of five to six "half-life" to reach stability condition. For example: If the medicine that the patient takes has a "half-life."

Equivalent to twelve hours as a "Lisinopril" (Prescription medication frequently to treat high blood pressure), the drug level stability in the blood case will start to downward gradually in half within sixty to seventy-two hours or a few days. In this case, blood pressure begins to rise as they have a negative impact on heart health. [1]

A recent medical study has revealed that patient's reminder text messages and dates medication can reduce the proportion of the deaths of thousands of patients, following a stroke or heart attack, as well as providing thousands of pounds. The study indicates that the omission of many patients for their medications cause loss of more than 500 million pounds, the value of the drugs provided by the National Health Service in Britain. It explained the new study, conducted by the University, "Queen Mary" in London, that the reminder text messages work to improve medication and absorption by $64 \%$, and not only works to maintain without wasting millions on prescription drugs not covered by the patients, but also prevents the death of many patients who need to take control of cholesterol drugs. The results obtained indicate that the patients' text messages to remind the dates of taking their medication is the most effective means of Statistics and the most effective way.[8]

The medicine drawers to organize patient's medications which easily founds in private pharmacies and actively helps if the patient forgets to eat their medications on a daily basis as they help patients who take more than one medication per day at different times.

Applicants age patients gain the most benefit from these drawers so that it is divided into several sections including covers a full week of medication (or more) as they are today divided into more than one period. These drawers can be carried easily in handbags while traveling, but patients have to be careful not to leave them in hot places (in the car, for example). [7]

There are many applications on smartphones, which help patients to remember pills time. For example, some free applications for smartphones, user can enter the dates of all the medicines that need to be addressed, the time and date. The Application will remind the user of the entire drugs, and it will also remind them of the date of their next visit to the doctor. It also saves all drugs covered or not covered. Patients can browse this information easily and refer to it at any time. [6]

Users can also add their feedback and can read more information about the medicine they take (such as side effects, different dosages, degree of safety for use during pregnancy and interactions with drugs or different foods). In some applications, users can also add pictures of their medicines if this method suits them best to remember their medication. All the information which users add to the application is stored on their phone only. This application is safe and guarantees the user's privacy.

\section{RELATED WORK}

Janckulik, D.; Martinovic, J.17-19 March 2009 an ad hoc system to monitor the ECG belted with a mobile phone in order to track the status of the patient from the beginning where the connection is through the Internet. [2]

Shaosheng Dai 2006 is a system that follows up the heart ICG planning and connects wireless directly with the patient in order to measure pressure, diabetes, heart rate. It also allows doctors to enter the application and the withdrawal of recorded data of the application to the patient's medical file and allows the application to conduct examination during daily life through the application. [4]

Sultan, S. 13-16 July 2009 is a system that works on mobile telephony delivers via Bluetooth, is working to develop some medical advice to patients. In addition, patients were directly connected with medical care center and interest application, primarily diabetes, and blood pressure. [5]

Shieh, S.C.; Lin, C.C.; Yang, T.F.; Tu, G.H. 8-11 Dec. 2008 infrared technology in pharmacy: It is a system based on the connection between the patients' PDA with compartments to organize the drug to be opened and closed using the contact infrared system RFID so as to prevent people from reaching the non-stating them medicine. [6]

Suzuki, T. 26-30 Aug. 2014 A smartphone mediated portable intelligent medicine case for medication management support is a system that works on smartphones that organizes taking medication schedules, such as birth control pills for women as well as he captures images of the medicine to be closets definition for each class of drug items in the application. The application calculates the daily doses as described by the doctor to the patient. [7]

Ermisoglu, E. 25-28 Aug. 2013 Simulation of Mobile Treatment Monitoring System, it shows that 50 to 75 of the patients forget the deadline for taking medication, so a system is developed that works on a mobile phone to organize appointments to make it easier for the users to take their medications on time from where they are connected with the Drawer for treatment. It directly helps the patient to know the medication required it was taken at the same moment. It is also useful for elderly patients. [8] 
Samir V. 11-12 December 2015 (IoT) Internet of Things network is considered as a health care system and as a home medicine reminder. It is a distant monitoring system, which gives real appointments of patient and health professionals. It is essential in connecting two technologies, which are: the use of messaging standard by Bluetooth or ZigBee and the use of communication reports. IOT stores sensors information, and it is essential for a quick reaction in case of emergencies. It is effective for people who have serious conditions and who need to take their pills on time, so it is recommended to use. RFID is an option for personal identification and for detecting objects. [9]

Abu-Dalbouh, Hussain 95-101 September a mobile reminder system is created to serve two necessary categories which are elderly and people who are diagnosed with Alzheimer. Both of these categories tend to forget things easily. This system can be used as their memories. It can be beneficial to them because it reminds them to take their medications on time, and due to that, they can take care of themselves. It also can be used as a food reminder. It provides real-time monitoring of direct provision of care and patient vital signs by using mobile telemedicine. [10]

Mobile-Health services and applications have become more popular these days. These services could save a lot of money because it can take care of the patients without going to the hospital every time. M-health applications are easy to use, which does not need any physical connection between patient and physicians. It is an effective way to enhance patient lives such as elderly, chronically ills, and disables. These health applications are not used for patients only, but essential for hospitals, care center. The fundamental role of these applications is to be used as reminder tools to improve medication adherence. [11]

Reddy and others proposed an intelligent system of drugs reminder for elderly patients. The system is capable of producing a buzzy sound and mention the drug name to the patient in the specified time. However, the system lacks the ability to change the drug name, and it is not connected to the patient mobile. [12]

Adabara used in his project an ATMEGA328/P microcontroller in implementing a circuit for patients' drugs reminder. A liquid crystal display with an alarm system is used to remind the patient with the time and name of the drug to be taken at a specific time. [13]

\section{HARDWARE EQUIPMENT}

Arduino is a great open-source project that aims to provide free software and coloring of the development of an interactive open-source Development Board used in the construction of electronic circuits smart and able to interact with humans and easily pleased. Anyone can use it to work legitimately own without the need for prior knowledge with the knowledge of electronics and without studying the intricacies of electronic circuits, and the painting is made up from the circle email with a microcontroller programmable through the Arduino IDE and software which can be downloaded free of charge for all operating systems. As shown in fig.1

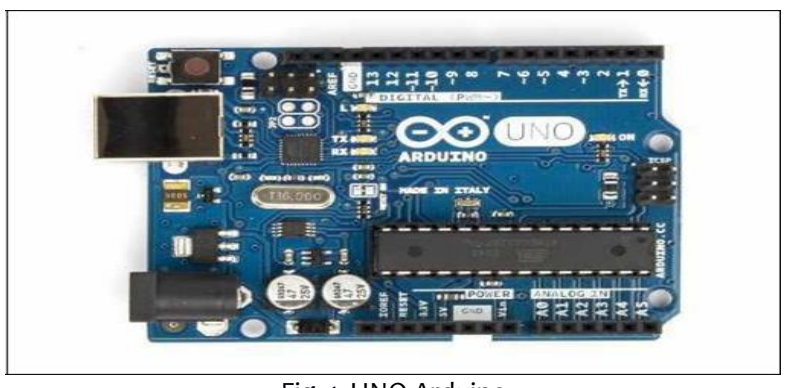

Fig. 1 UNO Arduino

Breadboard primarily to facilitate connectivity and installation of electronic circuits without the need for soldering process, where can accommodate many circuits that can connect the electricity regulator to electrical connections are not affected to conduct experiments and electronic test circuits. They offer a lot of efforts and time where the user can install circuit parts without soldering. There are many points that can be installed where the electronic circuit parts its divided so that every five points theoretically and practically one point. The breadboard used plate experiments to make temporary Electric circuits either to test the validity of connections or to experience the idea without the need to wild copper wires or components. Panel tests contain metal ports that allow the user to change any connections or the removal of any components easily, which allows using it over and over again.

Fig. 2 shows the Wi-Fi Arduino Shield. Wi-Fi shields its device to make a wireless connection between the iPhone and the Arduino board using 802.11 wireless specification which contains the pills drawers by using the static IP address as 192.168 .0 .200 by using long wire-wrap headers which extend through the shield.

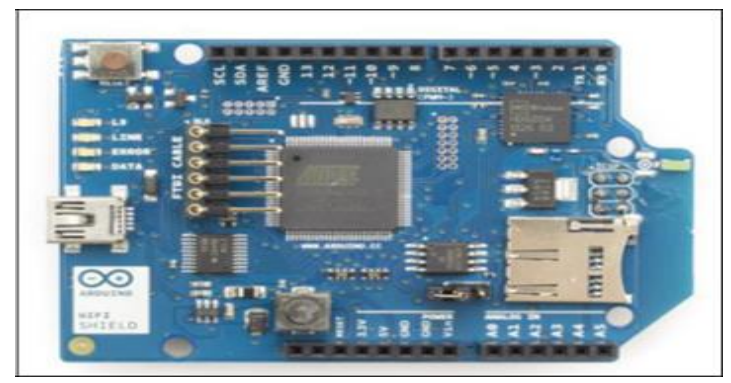

Fig. 2 Wi-Fi Arduino Shield

Micro Servo is a small motor used to open and shut Drawer medications. It is linked through Board Arduino. In this project, the Board Arduino has been linked with the application via Wi-Fi. 
Pills organizer is a small plastic box with different sizes can be carried. It usually contains seven drawers, according to the number of days of the week (divided from Saturday to the last day in the week, Friday). Also, the drawer has a brief to the days of labels, to facilitate the identification of the day and to identify the medication that must be addressed in this day. There are several designs of the drawers, this form that appears in the project has been chosen, for the ease of dealing with electronically, besides, it can be used to add some labels to let the user be more awareness to realize appointments.

\section{APPLICATION AND SYSTEM DESIGN}

The Application provides the user to set the timer to take medicine from the smart drawers. These drawers connect to the application wirelessly so; the application needs to connect to an internet network to run the features, and it only works in IOS devices. The application asks the user to be allowed to send the notification. Then, to make the communication between them, the user should click the "OK” button. If the user clicks "OK," the communication will start, but if the user clicks "Do not allow" the application will block the communication so the user will not send nor receive the notifications.

In case the user would like to receive a notification, the user can set and configure in the mobile settings. The home screen, which displays the list of the drawers in the application, the user can set the time for each drawer separately by click on the "set timer" button. To set the timer for the medicine, the user should open the pills drawer through the application to put the medicine by clicking the "Open" button. After taking medicine, the user can also close the drawer through the application by clicking the "Close" button, as shown in figure 8 . Then, the user will be moved to the "set timer" screen, as shown in fig. 3 .

In this screen Fig. 3, the user can scroll the time of the drawer to set the reminder. The application uses a 12-hour time format, and the user is allowed to set between AM and PM. After setting the time, the user must click the "Done" button to save changes then, click back.

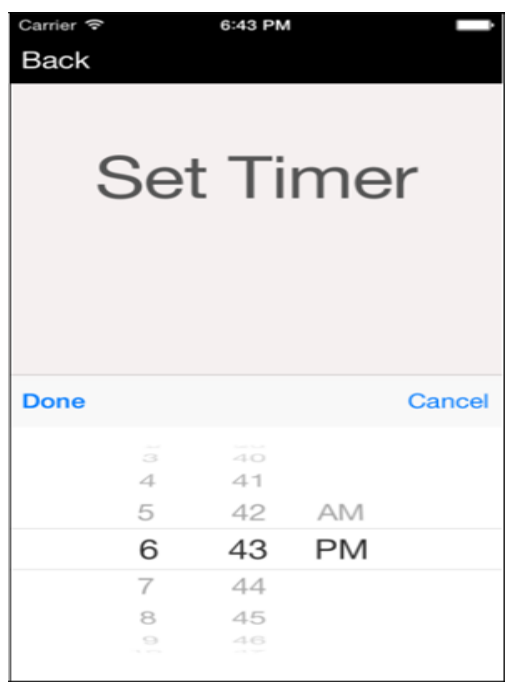

Fig. 3 Setting reminder time by the user

Fig. 4 shows the home screen after setting the timer for two drawers. The home screen contains the "settings" button that looks like the washer gear. After clicking the setting button in the upper right side, the user will be moved to the setting screen; to enter the settings of the application.

In "setting" screen, the user can add the IP address to connect between the Mobile app and the device by manual adding or automatic.

Fig. 5 shows the setting screen after adding the IP address. The User adds the IP 192.168.0.200 manually to connect the Smart Drawer.

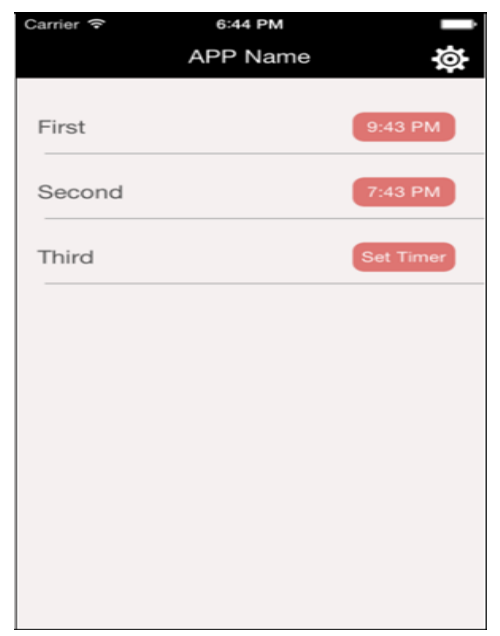

Fig. 4 Setting reminder time by the user 


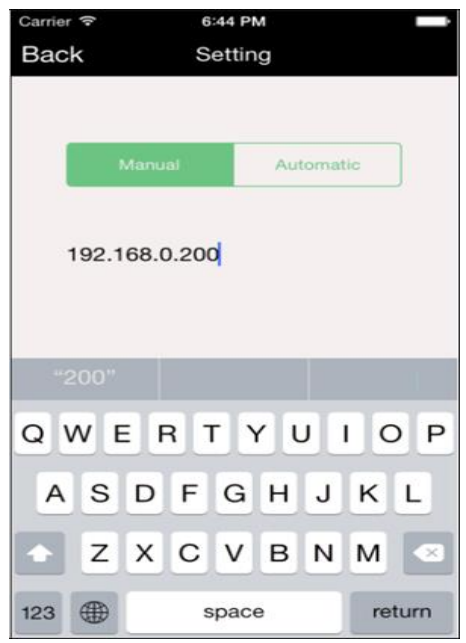

Fig. 5 Adding IP address manually

\section{APPLICATION AND SYSTEM DESING}

The Application, the Doses Organizer Application is one of the most important applications that increase a large number of users of IOS devices to regulate taking their medication schedules and remind them through the alarm to enable them to take their medication on time. The application's interface is clear and easy to use. It has an exquisite design and shows the user the types of medicine that are addressed. The application sends notifications when medicine time comes.

The application needs to connect to the internet network. One of the application's benefits is that user can open the daily Pharmaceuticals Drawer through the application and close it through either the application or manually and that for the safety of children of tampering with drugs and addressed.

\section{A. APPLICATION FEATURES:}

1. Regulates daily doses so that it appears on the screen the amount of medicine or the number of disks, the user will not be able to find difficulty in understanding the information.

2. The application alerts the user in a timely manner to take medicine according to the timing of the pre-set or pre-defined intervals.

3. User can specify a particular hour of taking the medication.

4. In case the user wants to determine the alarm clock to take medication at specific intervals, he can just set the starting hour and the length of time between the alarm and the next alarm.

5. The application removes the doses that have been dealt with automatically and will not be treading anymore whether the drug is covered or not.

6. Besides the drug and the dose name, the user can enter, any information associated with this medication as prescribed by the doctor.
7. The application can regulate eating more than one type of drugs at the same time.

8. The application maintains the confidentiality of the user and does not allow anyone tampers with the medicine which is in the drawers without accessing the application.

9. Application codifies what passed doses were taken from each of medicines is an essential record for those dealing drugs.

\section{B. APPLICATION LIMITATION:}

Limitations of any system or application are considered out of the question, each system or application in the first time has a few things missing, and that will be developed and fix it in the future. In this system, limitations can be summed in some of the following things:

1. The application supports only the English language.

2. The application supports only three drawers, and the user must repack it again.

3. The application requires an internet connection.

4. The application works on iPhone devices only.

\section{APPLICATION AND SYSTEM DESIGN}

The system allows communication between the application and the user through the interface of the application to use, where the user can choose all the parts through the buttons on the application. User can open drawers and shut down by the caller application completely connected through the Internet with smart drawers to conserve medications. That is easy to use in order to be a friend to all of the elderly who cannot cope with the complexity's techs.

Fig. 6 shows the connection between the application and the smart drawer. This connection needs an internet network. When the iPhone send signals to the Arduino Microcontroller while the iPhone is connecting to the internet, the user can open or close the smart drawer by clicking open/close buttons through the application

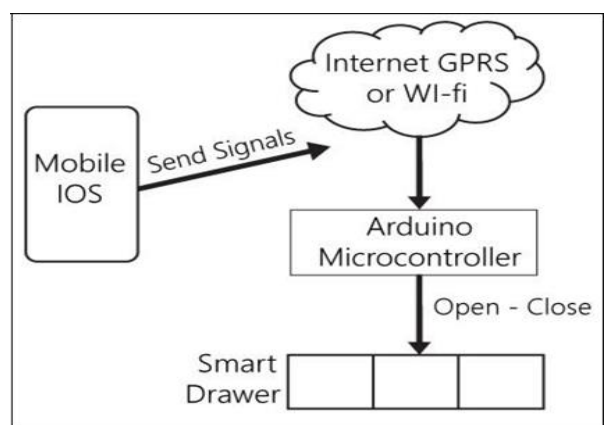

Fig. 6 Connection between the application and smart drawers 3

Fig. 7 displays a data flow diagram (DFD) shows how the system works. After installing the application and connecting the iPhone to the internet network to be 
contacted between the iPhone and pills drawer of the medicine, the user should allow the application to send notification (user can set that on the iPhone settings). Then, the user starts setting a timer for each pill's drawer, which is connecting to the application.

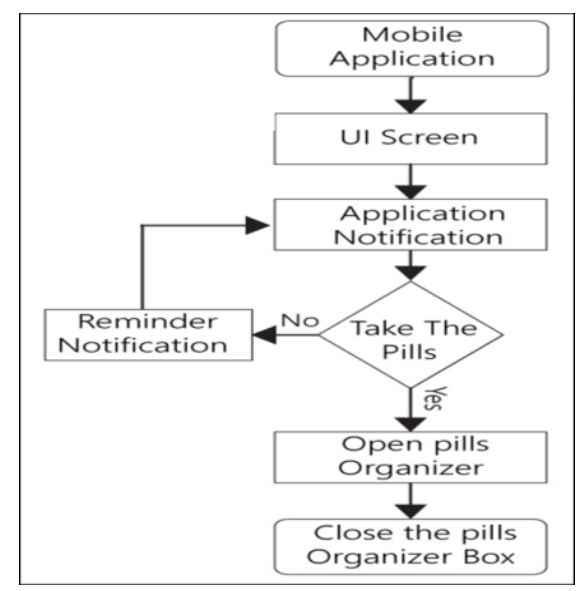

Fig. 7 How the system is work

When the user clicks the set timer button for any drawer, the user will be moved to a screen that provides the user to open the drawer by clicking the "Open" button. Therefore, the user can put the medicine on the drawer, and then the user can close the drawer through the application also by clicking the "Close" button. After that, the user set the timer by scrolling the time and then clicks the "Done" button

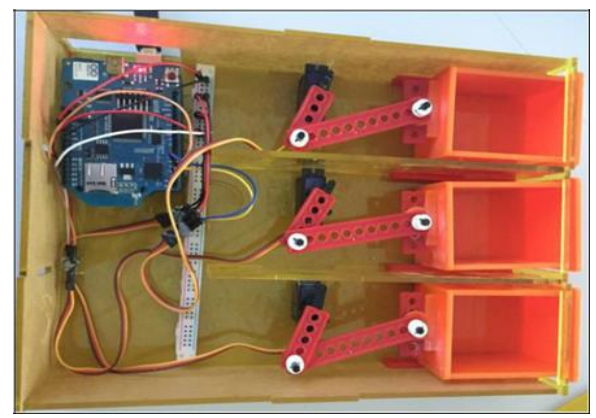

Fig. 8 The hardware of pills drawer

The connection between the mobile and the Arduino Shield Wi-Fi, the Wi-Fi is connected to the Arduino board, then it processes signals sent to it as shown in figure 8 . When the medicine time comes as the user set it, the application will send a notification to the user. In this case, the application will display a screen that contains two buttons (open and close buttons).

User clicks the "Open" button to open the drawer and take medicine. Then the application sends orders to Micro servo via the copper wires connected with a breadboard, and the micro servo pushes out the drawer the user to take medicine as shown in fig. 9.

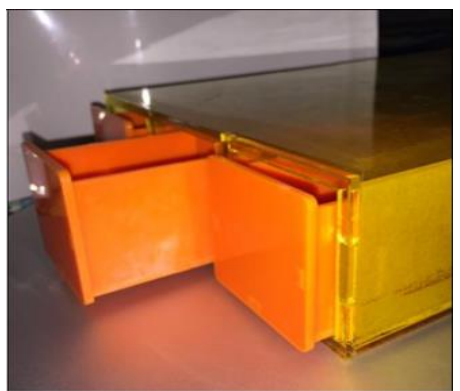

Fig. 9 Open pills drawer

To close the drawer after taking medicine, the user should click the "close" button. Also, the user can close the pills drawer either through the application (by "close" button) or manually.

\section{RESULT}

This paper presented an iPhone application that helps patients to organize and remember their medicine time. Besides, it provided a hardware system to connect the application with three smart drawers to store medicine with the ability to open and close these drawers by the application.

The application is easy to use; the screens are simple and clear to use. Users can set a timer for their medicine easily. However, the user may face a problem when the application was deleted; in this case, users have to return and enter all the data again. In addition, to change the language of the application, the user needs to change device language. Medical applications in this quality may be in the foreign store, but it is not available in the Arab Store.

In the future, many of the privileges will be added, and that will make a wide use for this application. Also, Drawer will be provided, and the Arabic will be reinforced. Also, the group looks forward, develops the application by calling the custom number when the user does not respond to the notification. As it will contain protection system, using highquality thumbs to enter the application.

\section{REFERENCES}

[1] W. Wai Yin, and Man Sang Wong. "Smart garment for trunk posture monitoring: A preliminary study." Scoliosis 3.7 (2008): 1-9.

[2] K. Ondrej, Dalibor Janckulik, and Jan Martinovic. "Smartphone, PDA and Embedded Devices as mobile monitoring stations of Biotelemetric System." GCC Conference \& Exhibition, 2009 5th IEEE. IEEE, 2009

[3] D. Gabriel J., et al. "A simple device to monitor flexion and lateral bending of the lumbar spine." NeuralSystems and Rehabilitation Engineering, IEEE Transactions on 13.1 (2005): 18- 23.

[4] D. Shaosheng, and Yue Zhang. "A wireless physiological multiparameter monitoring system based on mobile communication networks." Computer-Based Medical Systems, 2006. CBMS 2006. 19th IEEE International Symposium on. IEEE, 2006.

[5] Sultan. Salys, and Permanand Mohan. "How to interact: Evaluating the interface between mobile healthcare systems and the monitoring of blood sugar and blood pressure." Mobile and 
Ubiquitous Systems: Networking \& Services, MobiQuitous, 2009. MobiQuitous' 09. 6th Annual International. IEEE, 2009.

[6] S. Shien-Chii, et al. "Using RFID technology on clinic's pharmacy operation management and development of intelligent medicine dispensing cabinet." Industrial Engineering and Engineering Management, 2008. IEEM 2008. IEEE International Conference on IEEE, 2008.

[7] S. Takumi, and Yasushi Nakauchi. "A smartphone mediated portable intelligent medicine case for medication management support." Engineering in Medicine and Biology Society (EMBC), 2014 36th Annual International Conference of the IEEE, pp. 3642-3645. IEEE, 2014

[8] A. Idris, H. Edwards, and S. Mcdonald, "E-commerce adoption in Developing Countries SMEs: What Do the Prevailing E-commerce adoption in Developing Countries SMEs: What Do the Prevailing Theoretical Models Offer Us ?," no. October, 2017.

[9] E. Emre, Coskun Bayrak, and Engin Mendi. "Simulation of mobile treatment monitoring system." In Proceedings of the 2013 IEEE/ACM International Conference on Advances in Social Networks Analysis and Mining, pp. 978-981. ACM, 2013.
[10] Ermisoglu, Emre, Coskun Bayrak, and Engin Mendi. "Simulation of mobile treatment monitoring system." In Proceedings of the 2013 IEEE/ACM International Conference on Advances in Social Networks Analysis and Mining, pp. 978-981. ACM, 2013.

[11] Abu-Dalbouh, Hussain, Abrar Al-Habeeb, Atheer Al-Kholifi, Izdehar Al-Motiry, and Maryah Al-Buhairy. "A Mobile Reminder System for Elderly and Alzheimer's patients." International Journal of Computer Science Issues (IJCSI) 12, no. 5 (2015): 95

[12] Silva, Bruno MC, Joel JPC Rodrigues, Isabel de la Torre Díez, Miguel López-Coronado, and Kashif Saleem. "Mobile-health: A review of current state in 2015." Journal of biomedical informatics 56 (2015): 265-272.

[13] R. Surender Reddy, C. Niharika, Rohit Sharma, A. Bharath Kumar, and E.Narayana, "An Intelligent Patient Medicine Reminder System," International Journal of Innovative Research in Computer and Communication Engineering, Vol. 5, Issue 2, (2017): 2495- 2499.

[14] Adabara, I., "Patient Medication Reminder Circuit Using ATMEGA328/P Microcontroller: Design and Implementation," Mediterranean Journal of Basic and Applied Sciences (MJBAS), Vol. 2, Issue 1, (2018): 40-51. 